\title{
Experiments and micromechanical modeling of electrical conductivity of carbon nanotube/cement composites with moisture
}

\author{
Sung-Hwan Jang ${ }^{1,2}$, Daniel Peter Hochstein ${ }^{1,3}$, Shiho Kawashima ${ }^{1}$, Huiming Yin ${ }^{1}$ \\ ${ }^{1}$ Department of Civil Engineering and Engineering Mechanics, Columbia University, 610 \\ Seeley W. Mudd 500 West 120th Street, New York, NY 10027, USA \\ ${ }^{2}$ Robotics Institute, School of Computer Science, Carnegie Mellon University, Pittsburgh, PA \\ 15213, USA \\ ${ }^{3}$ Department of Civil and Environmental Engineering, Manhattan College, 4513 Manhattan \\ College Parkway, Riverdale, NY 10471, USA \\ * Corresponding author: Prof. Huiming Yin (yin@ civil.columbia.edu)
}




\begin{abstract}
Carbon nanotube (CNT)/cement composites have been proposed as a multifunctional material for self-sensing and traffic monitoring due to their unique electric conductivity which changes with the application of mechanical load. However, material constituent and environmental factors may significantly affect the potential application of these materials. Therefore, it is necessary to understand an influence of material constituent such as porosity and dispersion of CNT and environmental factor such as moisture on the electrical conductivity of CNT/cement composite. This paper investigates the effect of moisture on the effective electrical conductivity of $\mathrm{CNT} /$ cement composites. To prepare the specimens, multi-walled carbon nanotubes (MWCNTs) are well dispersed in cement paste, which is then molded and cured into cubic test specimens. By drying the specimens from the fully saturated state to the fully dry state, the effective electrical conductivity is measured at different moisture contents. As the water in the specimen is replaced by air voids, the electrical conductivity significantly decreases. Different ratios of MWCNTs to cement have been used in this study. Micromechanical models have been used to predict the effective electrical conductivities. A comprehensive model is proposed to take into account the effects of individual material phases on the effective electrical conductivity of $\mathrm{CNT} /$ cement composites with moisture effect.
\end{abstract}

Keywords: Electrical conductivity; cement paste; multi-walled carbon nanotubes; moisture; micromechanical model 


\section{Introduction}

Multifunctional cementitious-based composites using carbon nanotubes (CNTs) have attracted significant interest because of the improvement of their overall mechanical [1-4], electrical [5, 6], and thermal properties [7, 8]. Particularly, many researchers have conducted an electromechanical characterization of composites for sensing applications [9]. Azhari and Banthia [10] developed conductive cement-based composites using carbon fibers and carbon nanotubes, for application as a self-sensing material. Han et al. [11] investigated the piezoresistivity of multiwalled carbon nanotubes (MWCNTs)/cement composites as a function of the concentration of MWCNTs and the water-cement ratio for traffic monitoring applications.

The strain sensor using CNT dispersed composites is operated by a change in electrical resistance when it is subjected to mechanical loading such as compression or tension [12, 13]. The sensor resistance depends on the electrical conductivity and geometry of the composites in sensor fabrication. Therefore, knowing the electrical conductivity of the composite is important for sensor design. In general, there are three main factors that affect the electrical conductivity of cementitious-based composites containing MWCNTs. First, cement hydrate, as the matrix material, plays a role on the effective electrical conductivity of the composite although its electrical conductivity is quite low. Second, the voids in the matrix are filled with a combination of moisture and air, the voids will modify the effective electrical conductivity, which is very sensitive to ambient relative humidity, as the conductivity of liquid phase is much higher than that of air or cement hydrates in the composites [14, 15]. Third, MWCNTs also change the electrical conductivity of the composites due to their excellent electrical conductivity and the formation of a network of MWCNTs.

Since cementitious-based composites are typical heterogenoues materials with a significant amount of micro-voids and the dispersion of MWCNTs in these materials is random, a micromechanics-based approach is a useful tool to predict the effective material properties of the composite. For example, Princigallo et al. [16] studied the effect of the aggregate on the electrical conductivity of high-performance concretes. They investigated the properties of the interfacial zones by analyzing the electrical conductivity using several modeling approaches such as hard core soft shell model, differential effective medium theory, and the Lu-Torquato model. Schwarz et al. [17] employed a parallel model to predict the effective electrical conductivity as a 
function of the properties of the pore solution, the porosity, and also a pore connectivity factor. Liu et al. [18] proposed an analytical model which is a combination of effective medium and percolation theory to predict the relative resistivity of cement paste and C-S-H. While some experiments have demonstrated the effect of moisture or MWCNTs in cementitious-based composites $[19,20]$, it is still a challenging task to predict and model the effect of moisture and MWCNTs on the effective electrical conductivity of carbon nanotube/cement composites.

Mathematically, the effective electrical conductivity of a composite is a quantity exactly analogous to the effective dielectric permittivity, water permeability, and thermal conductivities, in a linear static state, so the modeling work can be conducted in the same fashion. Effective electrical properties of random composites have been widely studied by analytical, numerical, and experimental methods [21]. Maxwell-Garnett's model was one of the first to describe the effective permeability of composites containing randomly dispersed spherical particles [22, 23]. Because this model considers neither higher multipole moments nor interactions between particles, it generally underestimates the effective electrical conductivity when the particle permeability is higher than the matrix permeability, and vice versa, especially for composites with high volume fraction of particles [24]. Bruggeman extended Maxwell-Garnett's model by an iterative procedure and provided good agreement with experimental results. However, the effect of the microstructure has not been directly considered [25]. Yin and his colleagues have attempted to investigate the effective conductivity for particulate composites with some idealized microstructures $[26,27]$.

However, because CNT/cement composites exhibit such complex microstructures, which contain randomly dispersed and irregularly shaped voids that are filled by water or air and also curved MWCNTs, shown in Figure 1, none of micromechanic-based models can surely predict their effective electrical conductivities. Similarly to our previous work on viscoelastic behavior for asphalt mastics [28, 29], this paper will review existing micromechanics-based models, investigate the applicability of each model to the present composite through comparison with experiments, and thus establish an accurate formulation to predict the effective electrical properties of $\mathrm{CNT} /$ cement composites.

Three steps are considered to formulate the effective electrical conductivity of the composites based on the microstructure and size scales. In what follows, Section 2 will introduce the 
micromechanics-based models, which are generally developed for two-phase composites, and discuss the assumptions and applicability for each model. Section 3 will introduce an experimental procedure to measure the electrical conductivity of MWCNT/cement composites containing moisture. Section 4 will validate the formulation with experimental data, compare the models for the best performance, and thus establish a reliable model for the electrical conductivity of the CNT/cement composites. Some conclusive remarks will be provided in Section 5 .

\section{Micromechanics-based models for effective electrical conductivity of cement paste}

The micromechanics-based model was developed to estimate the overall material properties of composites. Various models are used to predict the effective electrical conductivity of CNT/cement composites, which is a quantity exactly analogous to effective dielectric permittivity, magnetic permeability, and thermal conductivity, in a linear static state [30]. For a multiphase composite containing discrete elements embedded in a continuous matrix, electrical conduction through the solid may depend on the properties of the individual material phases and its microstructure. The effective electrical conductivity can be obtained from the average of the local field in each material phase.

Consider a two-phase composite containing dispersions in a continuous matrix. To evaluate the effective electrical conductivity, the average electric field $\mathbf{E}$ and average current density $\mathbf{I}$ can be calculated from the volume averages of the two material phases as

$$
\begin{gathered}
\left\langle E_{i}\right\rangle_{D}=\phi\left\langle E_{i}\right\rangle_{\Omega}+(1-\phi)\left\langle E_{i}\right\rangle_{M} \\
\left\langle I_{i}\right\rangle_{D}=\phi\left\langle I_{i}\right\rangle_{\Omega}+(1-\phi)\left\langle I_{i}\right\rangle_{M}
\end{gathered}
$$

where the subscripts $\mathrm{D}, \Omega$, and $\mathrm{M}$ represent the composite, dispersed phase, and matrix phase, respectively; $\phi$ is the volume fraction of the dispersed phase; and each material phase is assumed to be isotropic satisfying

$$
\left\langle I_{i}\right\rangle_{\Omega}=\sigma_{\Omega}\left\langle E_{i}\right\rangle_{\Omega} \text { and }\left\langle I_{i}\right\rangle_{M}=\sigma_{M}\left\langle E_{i}\right\rangle_{M}
$$


in which $\sigma_{D}$ and $\sigma_{M}$ are the electrical conductivities of the two phases, respectively. The effective electrical conductivity can be calculated as

$$
\sigma_{e f f}=\frac{\left|\left\langle I_{i}\right\rangle_{D}\right|}{\left|\left\langle E_{i}\right\rangle_{D}\right|}=\frac{\phi\left\langle I_{i}\right\rangle_{\Omega}+(1-\phi)\left\langle I_{i}\right\rangle_{M}}{\phi\left\langle I_{i}\right\rangle_{\Omega} / \sigma_{\Omega}+(1-\phi)\left\langle I_{i}\right\rangle_{M} / \sigma_{M}}
$$

The parallel and series models provide the absolute upper and lower limits on the effective electrical conductivity of the composites. However, in general, the electrical conduction behavior of composites containing dispersed phases is far more complex than the above cases. Similarly to elastic modeling, in micromechanics to obtain the effective electric conductivity [27, 28], a uniform electric current density can be applied on the boundary of a composite and the local electrical fields will be disturbed by the material inhomogeneities. If the average electrical current on the dispersed phase can be obtained, from the above relation between the averaged electric field and the applied loading, the effective conductivity can be derived. The calculation is typically based on the solution for one particle, which may have more than one surface layer, embedded in a continuous matrix [31].

As in simple cases, one can assume the electric field or electric current density in both material phases are the same, which can mimic a laminate composite with the plane direction oriented parallel or perpendicular to the electric current direction, so that one can obtain the parallel and series models, respectively, as follows

$$
\begin{gathered}
\sigma_{e f f}^{\text {parallel }}=\phi \sigma_{\Omega}+(1-\phi) \sigma_{M} \\
\sigma_{e f f}^{\text {series }}=\frac{\sigma_{\Omega} \sigma_{M}}{\phi \sigma_{M}+(1-\phi) \sigma_{\Omega}}
\end{gathered}
$$

Consider a single spherical particle $\Omega$ embedded in a large continuous matrix phase $\mathrm{M}$ under a uniform electrical current density $\mathrm{I}^{0}$ in the far field. Due to the mismatch between the particle and the matrix, the local electric current density in the neighborhood of the particle will not be uniform any more. However, the electric current density on the particle is still uniform as [30]

$$
\mathrm{I}_{\Omega}=\frac{3 \sigma_{\Omega}}{\sigma_{\Omega}+2 \sigma_{M}} \mathrm{I}^{0}
$$


If the boundary conditions are given in terms of electrical potential or electric fields in the far field, the electrical field on the particle can be written as

$$
\mathrm{E}_{\Omega}=\frac{3 \sigma_{M}}{\sigma_{\Omega}+2 \sigma_{M}} \mathrm{E}^{0}
$$

In a general two-phase composite as Fig. 1(a), there are numerous dispersed particles and the microstructure varies randomly. It is formidable to formulate an exact local solution for a given composite sample. Fortunately, to obtain the effective electrical properties, only the averages of the electric field and current density are needed. Some homogenization methods are proposed to solve for the particle's average electrical field based on the above solution for one spherical particle embedded in the infinite domain. Different homogenization methods lead to different micromechanical models [28]. In what follows, we introduce five micromechanical models: the dilute model (DM), the Maxwell-Garnett model (MGM), the self-consistent model (SCM), the generalized self-consistent model (GSCM), and the differential scheme (DS), illustrated in Figs. $1(\mathrm{~b}-\mathrm{f})$, respectively.

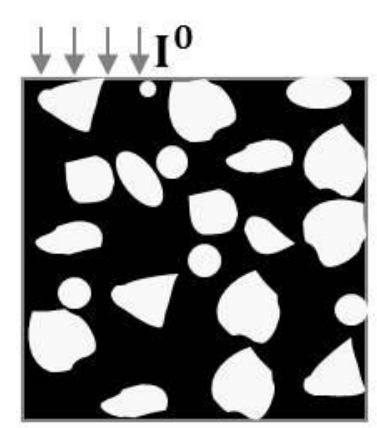

(a)

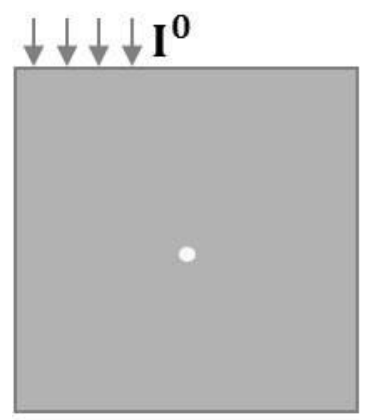

(d)

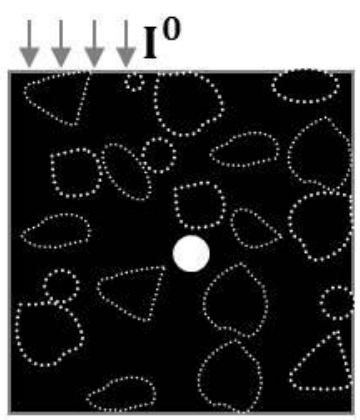

(b)

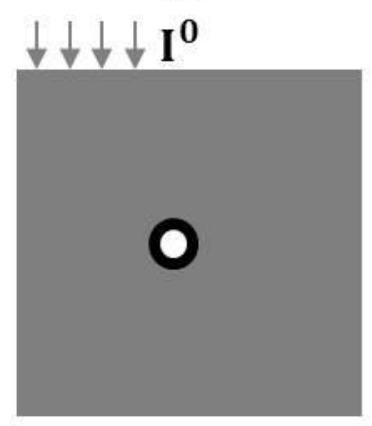

(e)

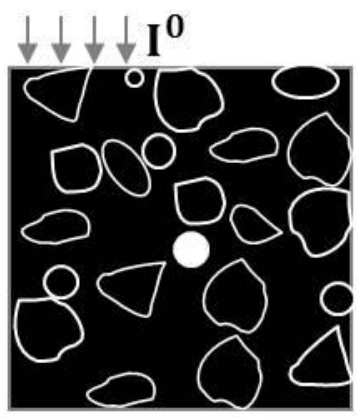

(c)

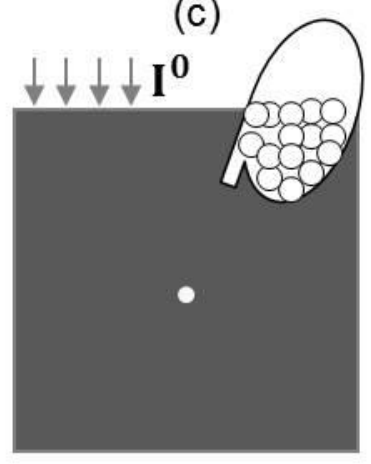

(f) 
Figure 1. A schematic illustration of micromechanical models: (a) Actual MWCNT/cement composite, (b) dilute model, (c) Maxwell-Garnett model, (d) Self-consistent model, (e) Generalized self-consistent model, and (f) Differential scheme

First, for the dilute model (DM), a dilute distribution of particles in a continuous matrix is considered. In Fig. 1(b), the effect of other particles is simply disregarded and the highlighted particle (white particle) is assumed to be spherical. Then, when an electrical current $I_{i}^{0}$ is applied in the far field, the particle averaged electrical current density can be obtained by one particle embedded in the matrix under the uniform loading. Obviously, the particle interaction and the effect of the particle shape are not considered. Therefore, this model is only applicable for composites with a very low volume fraction of spherical particles. The particle's averaged electrical current density can be directly obtained from the local solution in Eq. (7) as

$$
\left\langle I_{i}\right\rangle_{\Omega}=\frac{3 \sigma_{\Omega}}{\sigma_{\Omega}+2 \sigma_{M}} I_{i}^{0}
$$

Because the average electrical current density should be consistent with the applied one, i.e. $\left\langle I_{i}\right\rangle_{\Omega}=I_{i}^{0}$, the combination of Eq. (9) with Eqs. (1) - (4) yields

$$
\sigma_{e f f}^{D M-I}=\sigma_{M} \frac{\sigma_{\Omega}+2 \sigma_{M}}{\left(\sigma_{\Omega}+2 \sigma_{M}\right)-3 \phi\left(\sigma_{\Omega}-\sigma_{M}\right)}
$$

Similarly, if the boundary conditions are given in terms of electrical potential or electric fields in the far field, one can write

$$
\left\langle E_{i}\right\rangle_{\Omega}=\frac{3 \sigma_{M}}{\sigma_{\Omega}+2 \sigma_{M}}\left\langle E_{i}\right\rangle_{D}
$$

and then the effective electrical conductivity can be written as

$$
\sigma_{e f f}^{D M-E}=\sigma_{M}+\phi \frac{3 \sigma_{M}\left(\sigma_{\Omega}-\sigma_{M}\right)}{\sigma_{\Omega}+2 \sigma_{M}}
$$


Because the dilute distribution of particles is assumed, this model is only valid for composites with very small volume fractions of dispersed phases. Based on two different boundary conditions, two predictions are obtained in Eqs. (10) and (12). Obviously, they are close to each other when the volume fraction of particles is very small. However, when the volume fraction increases to a certain value, they are different because the one particle solution is not applicable any more. The results of Eq. (10) can be divergent because the denominator in the equation may reduce to zero (for instance, as the volume fraction $\phi=\left(\sigma_{\Omega}+2 \sigma_{M}\right) /\left(\sigma_{\Omega}-\sigma_{M}\right) / 3$, Eq. (10) is found to diverge).

Secondly, for the Maxwell-Garnett model (MGM) [22], one can derive it in a straightforward method. A particle is embedded into the matrix with a uniform electric current density the same as the matrix's averaged electric current density. In Figure 1 (c), before the highlighted particle is embedded in the matrix, due to the existence of other particles, the electrical current density field surrounding the highlighted particle is highly distorted. The MGM assumes the electrical current density in the neighborhood of the particle is equal to the matrix's averaged electric current density. When the particle is embedded in the matrix, the particle averaged electrical field can be obtained by considering one particle embedded in the matrix with a uniform current density. The particle's averaged electric current density is written as

$$
\left\langle I_{i}\right\rangle_{\Omega}=\frac{3 \sigma_{\Omega}}{\sigma_{\Omega}+\sigma_{M}}\left\langle I_{i}\right\rangle_{M}
$$

The substitution of Eq. (13) into Eq. (4) yields

$$
\sigma_{e f f}^{M G M}=\sigma_{M} \frac{\left(\sigma_{\Omega}+2 \sigma_{M}\right)+2 \phi\left(\sigma_{\Omega}-\sigma_{M}\right)}{\left(\sigma_{\Omega}+2 \sigma_{M}\right)-\phi\left(\sigma_{\Omega}-\sigma_{M}\right)}
$$

Similarly, if the particle's average electrical field is considered as $\left\langle E_{i}\right\rangle_{\Omega}=\frac{3 \sigma_{M}}{\sigma_{\Omega}+2 \sigma_{M}}\left\langle E_{i}\right\rangle_{M}$, one can also derive the effective electrical conductivity, which is the same as the above.

When the volume fraction of particles is fairly high or particles are clustered, even in the neighborhood of the particle other particles may exist, so the local field in the particle domain can interact with other particles. Consequently, although MGM provides a better prediction than 
DM for most composites, for high volume fractions or a clustered microstructure, it produces a considerable disparity from the experimental results. Yin and Sun [30] considered the pairwise interaction of randomly dispersed particles and made some improvements. In addition, in the MGM, the particle is still assumed to be spherical, so the effect of the particle shape is not considered. Notice that the MGM model is equivalent to the Hashin and Shtrikman lower bounds for $\sigma_{\Omega}>\sigma_{M}$ [32]. If we switch the material phases, we can similarly obtain the Hashin and Shtrikman upper bounds as follows:

$$
\sigma_{e f f}^{H S U}=\sigma_{\Omega} \frac{\left(\sigma_{M}+2 \sigma_{\Omega}\right)-2(1-\phi)\left(\sigma_{\Omega}-\sigma_{M}\right)}{\left(\sigma_{M}+2 \sigma_{\Omega}\right)+(1-\phi)\left(\sigma_{\Omega}-\sigma_{M}\right)}
$$

Thirdly, for the self-consistent method (SCM), because the microstructure is statistically random, the averaged electrical current density of the particle phase is represented by that of a randomly chosen infinitesimal material point embedded in the composite itself, as illustrated in Figure 1(d). Because the point is so small, its effect on the effective material properties of the remaining composite is negligible. Therefore, the particle's average electrical current density can be obtained by a single infinitesimal particle embedded in the composite itself under the uniform loading as follows:

$$
\left\langle I_{i}\right\rangle_{\Omega}=\frac{3 \sigma_{\Omega}}{\sigma_{\Omega}+2 \sigma_{e f f}^{S C M}}\left\langle I_{i}\right\rangle_{D}
$$

Because the point can be chosen at any location in the particle phase, this model is applicable for general composites with irregular shapes and a high volume fraction of particles. The combination of Eq. (16) with Eqs. (1) - (4) yields

$$
\sigma_{e f f}^{S C M}=\frac{1}{\phi \frac{3}{\sigma_{\Omega}+2 \sigma_{e f f}^{S C M}}+\left[1-\frac{3 \phi \sigma_{\Omega}}{\sigma_{\Omega}+2 \sigma_{e f f}^{S C M}}\right] / \sigma_{M}}
$$

Simplifying the above equation, the effective electric conductivity can be explicitly solved as

$$
\sigma_{\text {eff }}^{S C M}=\frac{-\alpha+\sqrt{\alpha^{2}+8 \sigma_{\Omega} \sigma_{M}}}{4}
$$


where $\alpha=(1-3 \phi) \sigma_{\Omega}+(3 \phi-2) \sigma_{M}$.

Fourthly, for the generalized self-consistent model (GSCM), as shown in Fig. 1(e), the particle's average electrical current field is derived from the solution for a spherical particle embedded in a concentrical spherical annulus of the matrix of the prescribed volume fraction, which in turn is embedded in an infinite medium with the effective electric properties of the matrix [31]. Therefore, the effective electrical conductivity of the composite can be obtained according to the concept of GSCM as: [32, 33]

$$
\sigma_{e f f}^{G S C M}=\sigma_{M} \frac{\left(\sigma_{\Omega}+2 \sigma_{M}\right)+2 \phi\left(\sigma_{\Omega}-\sigma_{M}\right)}{\left(\sigma_{\Omega}+2 \sigma_{M}\right)-\phi\left(\sigma_{\Omega}-\sigma_{M}\right)}
$$

Because the neighboring material plays a more important role on the local electric field of a particle than the far field material does, GSCM uses the solution for a double layered particle embedded in the composite itself to obtain the particle averaged electric field. Compared to the self-consistent method, although the local interaction between the particle and the matrix is considered, the assumption of the spherical shape of the particle and the matrix layer is imposed, which is not always valid for general composites. Mathematically, it provides the same prediction as the MGM model in Eq. (14). Therefore, we will not individually consider it in the following part of this paper.

Fifthly, the differential scheme (DS) was proposed by the observation that a composite with a finite volume fraction of particles can be fabricated through the process of adding more and more particles in Fig. 1(f). Start with the pure matrix with $\sigma_{M}$ and volume $V_{0}$, add a small volume of particle $V_{1}$ into the matrix for a volume fraction $\phi_{1}=\frac{V_{1}}{V_{0}+V_{1}}$, the particle's average electrical field can be obtained from the above the dilute model as

$$
\left\langle E_{i}\right\rangle_{V_{1}}=\frac{3 \sigma_{M}}{\sigma_{\Omega}+2 \sigma_{M}}\left\langle E_{i}\right\rangle_{D}
$$

The average electric field is 


$$
\left\langle E_{i}\right\rangle_{D}=\phi_{1} \frac{3 \sigma_{M}}{\sigma_{\Omega}+2 \sigma_{M}}\left\langle E_{i}\right\rangle_{D}+\left(1-\phi_{1}\right)\left\langle E_{i}\right\rangle_{M}
$$

In consequence, one can obtain

$$
\left\langle E_{i}\right\rangle_{M}=\frac{1}{1-\phi_{1}}\left(1-\phi_{1} \frac{3 \sigma_{M}}{\sigma_{\Omega}+2 \sigma_{M}}\right)\left\langle E_{i}\right\rangle_{D}
$$

and

$$
\left\langle I_{i}\right\rangle_{D}=\phi_{1} \frac{3 \sigma_{M}}{\sigma_{\Omega}+2 \sigma_{M}}\left\langle E_{i}\right\rangle_{D} \sigma_{\Omega}+\left(1-\phi_{1}\right) \frac{1}{1-\phi_{1}}\left(1-\phi_{1} \frac{3 \sigma_{M}}{\sigma_{\Omega}+2 \sigma_{M}}\right)\left\langle E_{i}\right\rangle_{D} \sigma_{M}=\left\langle E_{i}\right\rangle_{D}\left[\frac{3 \phi_{1} \sigma_{M} \sigma_{\Omega}}{\sigma_{\Omega}+2 \sigma_{M}}+\left(1-\phi_{1} \frac{3 \sigma_{M}}{\sigma_{\Omega}+2 \sigma_{M}}\right) c\right.
$$

Therefore, we obtain the incremental of the effective electric conductivity is

$$
\begin{aligned}
& \bar{\sigma}_{e f f}^{0}=\sigma_{M}+\sigma_{M}\left[3 \phi_{1} \frac{\sigma_{\Omega}-\sigma_{M}}{\sigma_{\Omega}+2 \sigma_{M}}\right] \\
& \Delta \bar{\sigma}_{e f f}^{0}=\phi_{1} \frac{3 \sigma_{M}}{\sigma_{\Omega}+2 \sigma_{M}}\left(\sigma_{\Omega}-\sigma_{M}\right)
\end{aligned}
$$

Treat the new composite as a matrix with $\bar{\sigma}_{\text {eff }}$, add another small volume of particles, and update the volume fraction and effective electrical conductivity. Step by step, one can cumulate the overall volume fraction of the particles to a finite number less than 100\%. From step $i$ to step $i+1$, the volume of the particle increases from $V_{1}$ to $V_{i+1}$ with an increment $\Delta V_{i}$, so the volume fractions at the two steps are written

$$
\phi_{i+1}=\frac{V_{i}+\Delta V_{i}}{V_{0}+V_{i}+\Delta V_{i}} ; \phi_{i}=\frac{V_{i}}{V_{0}+V_{i}}
$$

The volume fraction increment is written

$$
\Delta \phi_{i}=\phi_{i+1}-\phi_{i}=\left(1-\phi_{i}\right) \frac{\Delta V_{i}}{V_{0}+V_{i}+\Delta V_{i}}
$$


At step $i$, the effective conductivity is written $\bar{\sigma}_{\text {eff }}^{i}$. With the incremental volume of particles, similarly to Eq. (10), one can

$$
\Delta \bar{\sigma}_{e f f}^{i+1}=\frac{\Delta V_{i}}{V_{0}+V_{i}+\Delta V_{i}} \frac{3 \bar{\sigma}_{e f f}^{i}}{\sigma_{\Omega}+2 \bar{\sigma}_{e f f}^{i}}\left(\sigma_{\Omega}-\bar{\sigma}_{e f f}^{i}\right)=\frac{\Delta \phi}{1-\phi_{i}} \frac{3 \bar{\sigma}_{e f f}^{i}}{\sigma_{\Omega}+2 \bar{\sigma}_{e f f}^{i}}\left(\sigma_{\Omega}-\bar{\sigma}_{e f f}^{i}\right)
$$

Considering $\Delta V_{i}$ can be an infinitesimal value, the above equation can be rewritten in the differential form as

$$
\frac{d \sigma_{e f f}}{d \phi}=\frac{1}{1-\phi} \frac{3 \sigma_{e f f}}{\sigma_{\Omega}+2 \sigma_{e f f}}\left(\sigma_{\Omega}-\sigma_{e f f}\right)
$$

When $\phi=0, \sigma_{\text {eff }}=\sigma_{M}$. Therefore, one can write the effective electrical conductivity as

$$
\sigma_{\text {eff }}=\sigma_{\Omega}\left\{\left[\sqrt{\left(\frac{b}{3}\right)^{3}+\left(\frac{b}{2}\right)^{2}}-\left(\frac{b}{2}\right)\right]^{1 / 3}-\frac{b}{3}\left[\sqrt{\left(\frac{b}{3}\right)^{3}+\left(\frac{b}{2}\right)^{2}}-\left(\frac{b}{2}\right)\right]^{-1 / 3}\right\}+\sigma_{\Omega}
$$

where $b=(1-\phi)^{3}\left(1-\sigma_{M} / \sigma_{\Omega}\right)^{3} \frac{\sigma_{\Omega}}{\sigma_{M}}$.

Notice that the differential scheme is a general approach and can be applicable to different micromechanical models. In the above, each infinitesimal incremental step essentially uses the dilute model of Eq. (12) and overall an explicit analytical solution is provided in Eq. (30). If other models are used, different predictions may be obtained. For example, if Eq. (10) is used, one can replace $1 / \sigma$ as a new variable, say resistance, to set up the differential equation and eventually to calculate the effective electrical conductivity, which is the same as Eq. (30). However, when other models are used, analytical formulation may not exist but numerical solution will be possible.

\section{Experiments}

\subsection{Materials and sample preparation}


Type I ordinary Portland cement was used from Lafarge. Table 1 shows the chemical properties of the cement. Pristine MWCNTs were purchased from Nanolab, USA as chemically treated MWCNTs may hamper the hydration of cement in the curing process [34]. The commercial MWCNT had a carbon purity $>85 \%$, an average diameter of $20.0 \mathrm{~nm}$, and length of $12.5 \mu \mathrm{m}$, as shown in Table 2. Figure 2 showed SEM image of as-received MWCNTs where lots of agglomeration exists due to the van der Waals interaction [35].

Table 1. Chemical composition of cement

\begin{tabular}{|c|c|c|c|}
\hline \multicolumn{2}{|c|}{ Oxide Composition (wt.\%) } & \multicolumn{2}{|c|}{ Mineralogical phase composite (wt.\%) } \\
\hline $\mathrm{CaO}$ & 63.7 & $\begin{array}{l}\text { Tricalcium silicate } \\
\left(\mathrm{C}_{3} \mathrm{~S}\right)\end{array}$ & 52.4 \\
\hline $\mathrm{SiO}_{2}$ & 12.9 & $\begin{array}{l}\text { Dicalcium silicate } \\
\left(\mathrm{C}_{2} \mathrm{~S}\right)\end{array}$ & 16.0 \\
\hline $\mathrm{Fe}_{2} \mathrm{O}_{3}$ & 7.9 & $\begin{array}{l}\text { Tricalcium aluminate } \\
\left(\mathrm{C}_{3} \mathrm{~A}\right)\end{array}$ & 10.2 \\
\hline $\mathrm{SO}_{3}$ & 5.3 & $\begin{array}{l}\text { Tetra-calcium } \\
\text { Aluminoferrite } \\
\left(\mathrm{C}_{4} \mathrm{AF}\right)\end{array}$ & 8.6 \\
\hline $\mathrm{Al}_{2} \mathrm{O}_{3}$ & 4.2 & Magnesite & 0.3 \\
\hline $\mathrm{MgO}$ & 3.5 & Calcite & 0.5 \\
\hline $\mathrm{K}_{2} \mathrm{O}$ & 0.9 & Dolomite & 1.2 \\
\hline $\mathrm{TiO}_{2}$ & 0.3 & & \\
\hline $\mathrm{ZnO}$ & 0.2 & & \\
\hline
\end{tabular}

Table 2. Physical properties of MWCNTs

\begin{tabular}{ll}
\hline Properties & Values \\
\hline Density & $1.8 \mathrm{~g} / \mathrm{cm}^{3}$ \\
Surface area & $250-300 \mathrm{~m}^{2} / \mathrm{g}$
\end{tabular}


Diameter

Length

Electrical conductivity

Purity
$10-30 \mathrm{~nm}$

$5-20 \mu \mathrm{m}$

$1 \times 10^{5} \mathrm{~S} / \mathrm{m}$

$>85.0$ wt. $\%$

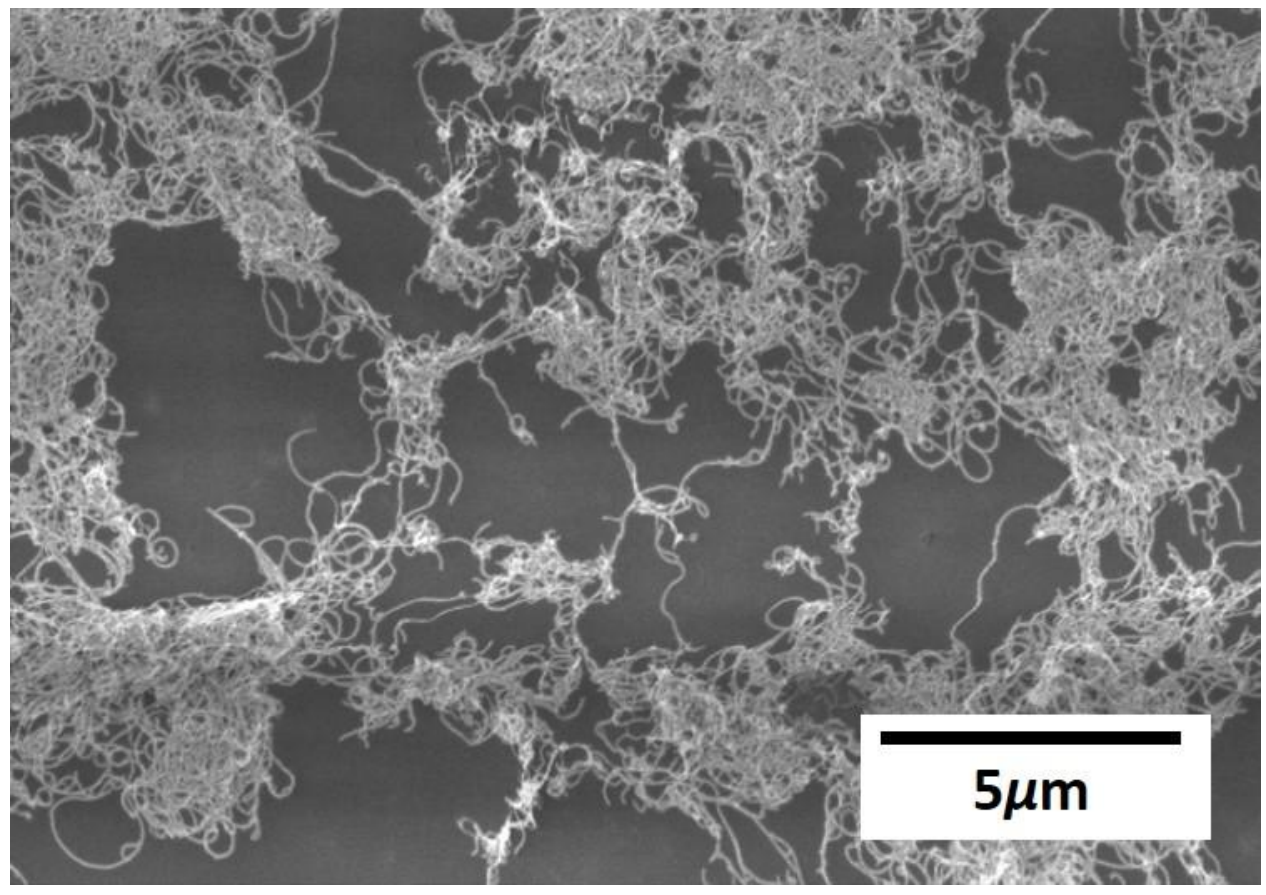

Figure 2. SEM image of dispersed MWCNTs in water

The uniform dispersion of MWCNTs is very important in preparing conductive materials. In this study, MWCNTs were dispersed in distilled water using a horn-type ultrasonication (Fisher Scientific, USA) for $30 \mathrm{~min}$. on a pulse mode ( $20 \mathrm{sec}$. on / $20 \mathrm{sec}$. off). A total energy of 20,000J was used in ultrasonication for each specimen. The suspension of MWCNTs in water was kept in an ice bath during the sonication to prevent damage from the heat. After the sonication, the cement was mixed with the MWCNT suspension $(0,0.05,0.10,0.50$, and $1.00 \%$ by cement weight) for about $5 \mathrm{~min}$. After pouring the mixture into cubic molds with the edge length of 25.4 $\mathrm{mm}$, vibration was applied for compaction and densification of the cement paste. All specimens were demolded in $24 \mathrm{hrs}$. Then, the specimens were cured in a water bath at room temperature 
for 28 days. After the specimens were ready for tests, different moisture conditions were applied for the electrical characterization.

\subsection{Characterization}

The distribution of MWCNTs in cement hydrates was observed using a scanning electron microscope (JEOL SEM-6400). After 28 days, the specimens were weighed before heating them in an oven at $100 \pm 5{ }^{\circ} \mathrm{C}$ for a specific period of time in order to produce different moisture contents. The moisture content of a specimen, $w$, is calculated as

$$
w=\frac{w_{t}-w_{d}}{w_{d}} \times 100(\mathrm{wt} . \%)
$$

where $w_{t}$ is the weight of the specimen to be evaluated and $w_{d}$ is the weight of the specimen after a full drying process.

The two point-probe method was employed to measure the volume resistance due to difficulty in installing electrodes inside cement paste as well as the oxidation of electrodes in the four point probe method [36, 37]. The volume resistance of the specimens was measured using a Fluke 8846A multimeter at room temperature. Silver paint was applied at both electrodes of the specimen to reduce the contact resistance between the test probe and the sample surface. Notice that because the contact resistance is much smaller than the resistance of samples, the advantages of the four point-probe method over the two-probe method are not significant. A customized test fixture with copper plates was also used to measure the resistance of the specimens under the same conditions in terms of the pressure and the temperature. The electrical conductivity $(\sigma)$ of the specimens was calculated from the bulk resistance by

$$
\sigma=\frac{L}{R A}
$$

where $L$ is the length between the electrodes, $A$ is the cross-sectional area of the specimens, and $R$ is the volume resistance. Figure 3 showed the sample preparation and the experimental setup for the electrical conductivity of the composites. 


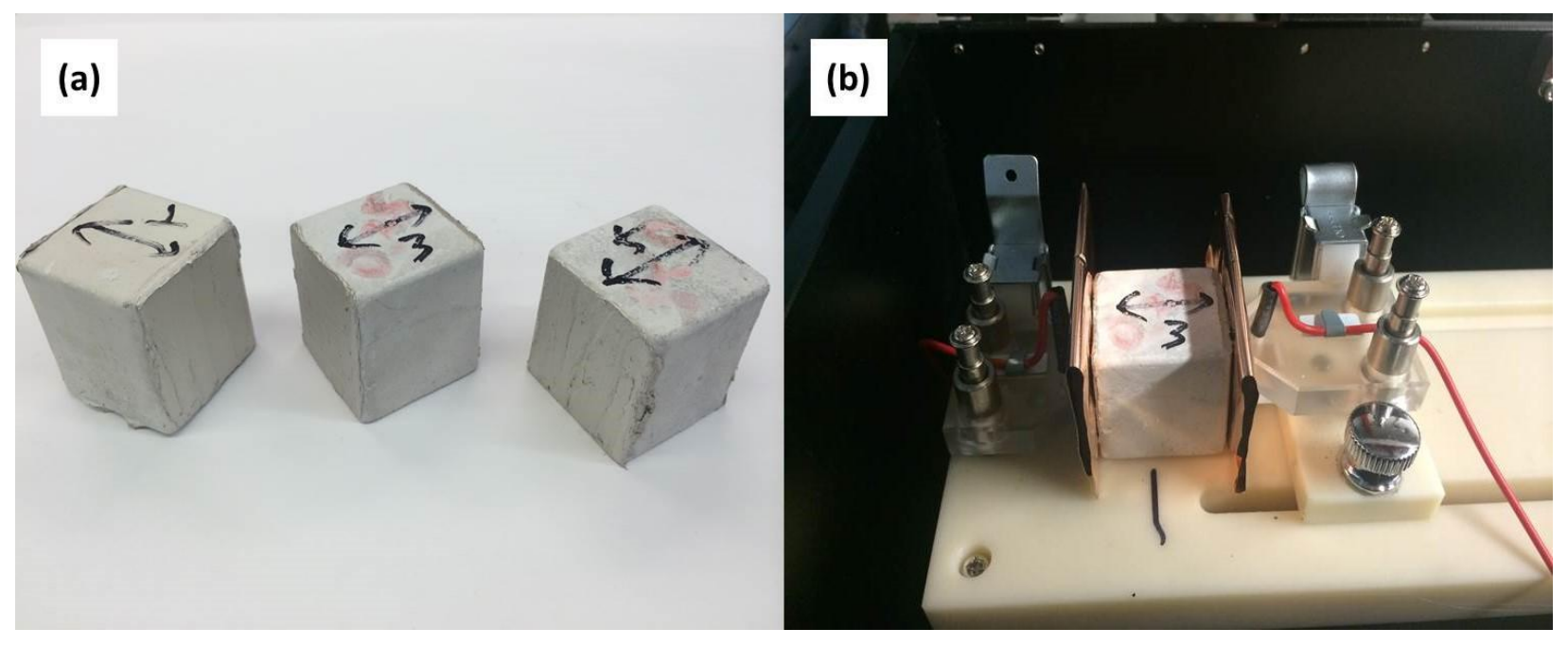

Figure 3. Volume resistance measurement of MWCNT/cement composite specimens. (a) Silver painted specimens and (b) Setup for resistance measurement

\section{Results and discussion}

\subsection{Microstructure of the MWCNT/cement paste}

Although many researchers have proposed different dispersion methods for MWCNTs in cementitious-based composites, it is still a challenging task due to the polarity of water and the alkalinity of cement [38, 39]. This explains the agglomeration of MWCNTs that has been observed in this study. Figure 4 shows the dispersion of MWCNT in cement hydrates (figure 4(a)) and highlights an area with a red dash line where many MWCNTs have agglomerated (figure 4(b)). This non-uniform distribution of MWCNTs may significantly reduce the overall electrical conductivity of MWCNT/cement paste composites. In this study, we consider different phases such as the cement hydrate, air voids, MWCNTs, and moisture in order to predict the effective electrical conductivity of MWCNT/cement paste. First, one can simulate cement paste prepared at different porosities by using a two-phase model with one phase being the cement hydrates and the other being the pores. Then, moisture is introduced into the composite to investigate the effect of moisture on electrical conductivity of MWCNT/cement paste. By considering the cement paste as a uniform matrix and introducing MWCNTs into the matrix, one can also 
simulate MWCNT/cement paste. Finally the combination of the three steps will provide a prediction to the effective conductivity giving the porosity, moisture content, and MWCNT content.

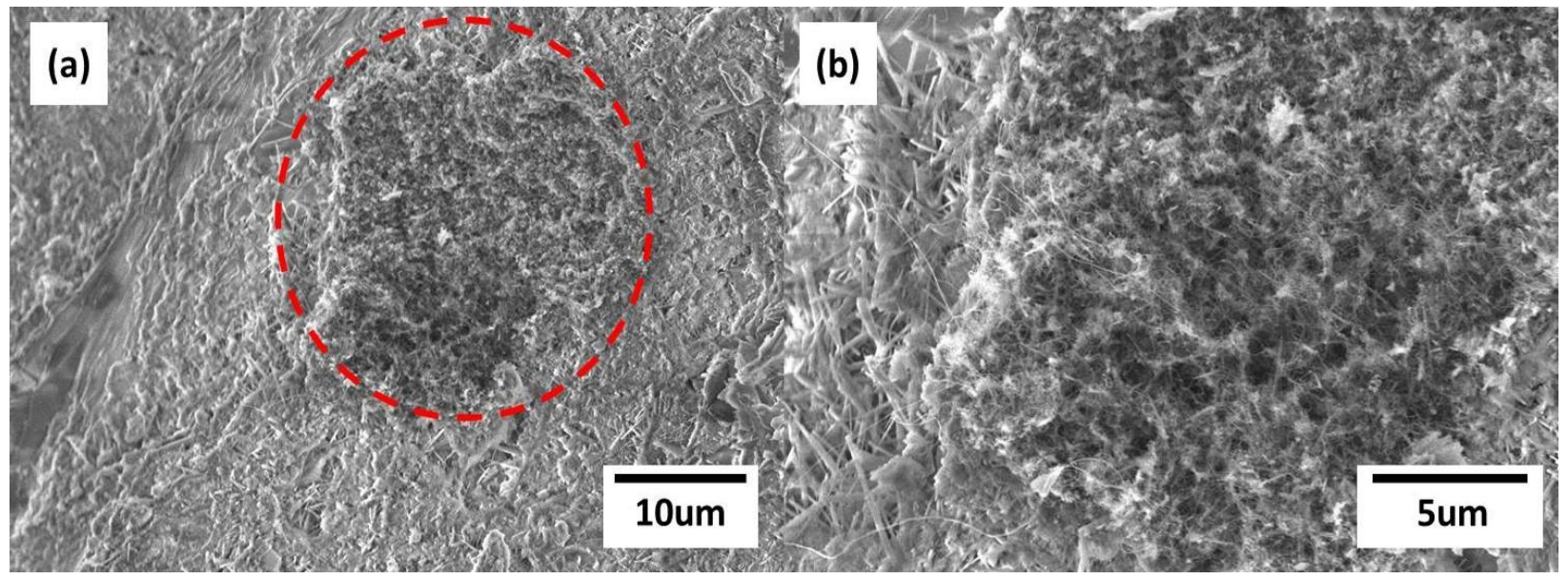

Figure 4. MWCNT agglomeration in cement hydrates

\subsection{Comparison of micromechanical models}

Overall, because MWCNTs as well as the moisture contained in the cement hydrates are generally irregular shapes such as angular, flat, or sub-rounded, whereas DM, MGM, and GSCM are based on the assumption of spherical particles, the accuracy of these models is questionable. Although SCM does not consider the interaction between the cement hydrates and the MWCNTs as well as moisture, it offers some flexibility for the particle shape and volume fraction. It is noted that all these models are based on the solution for one spherical particle embedded in an infinite domain, so the direct interaction between particles is not considered. Figure 5 shows a comparison of various models for the relative effective electrical conductivity $\left(\sigma_{\text {filler }} / \sigma_{\text {matrix }}\right)$. As expected, the Parallel and Series models provide the highest and lowest values, respectively and the DM also predicts low values as well. Out of the DS, SCM, MGM, and GSCM models, the MGM provides the lowest prediction whereas the SCM shows the highest prediction. At low volume fraction, there is no significant difference between the DM, MGM, SCM, GSCM, and 
DS models, regardless of the conductivity of fillers. Because the Parallel and Series only provide a large range of the upper and lower bounds instead of the specific predictions of the effective electrical conductivities, we will not include them the following comparison.

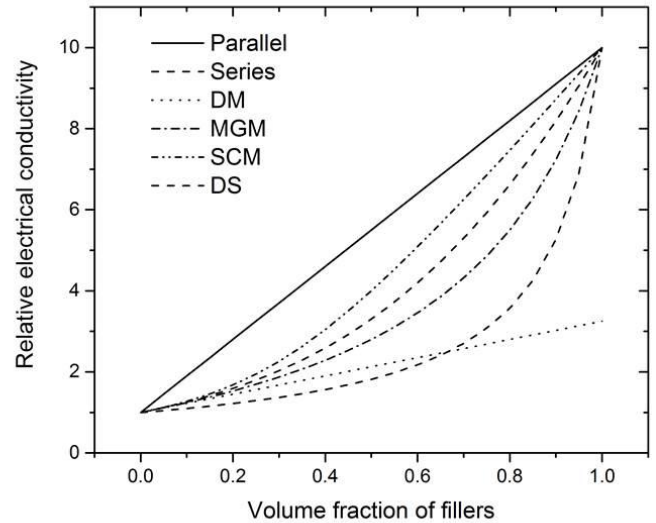

(a)

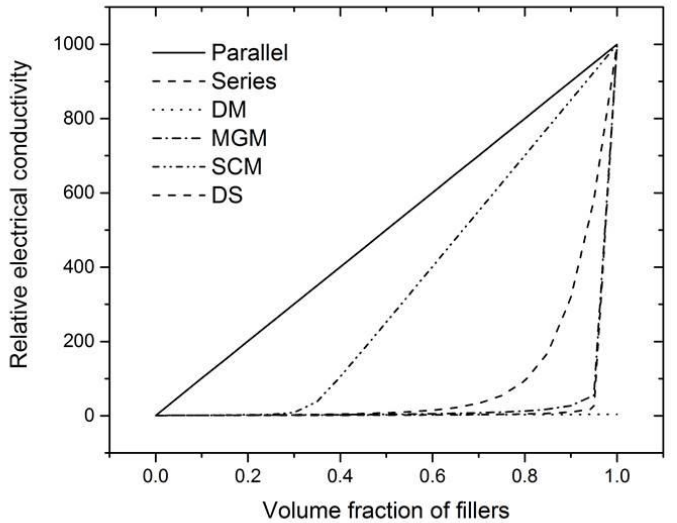

(b)

Figure 5. Comparison of various models for the effective electrical conductivity (a)

$$
\sigma_{\text {filler }} / \sigma_{\text {matrix }}=10 \text { and (b) } \sigma_{\text {filler }} / \sigma_{\text {matrix }}=1,000
$$

\subsection{Influence of porosity in cement paste}

The electrical conductivity of cement paste is associated with the pore structure, which is related to the water-to-cement ratio. The porosity of cement paste as a function of the water-to-cement ratio was obtained by Hansen's model [40] :

$$
\phi_{p}=\frac{w / c-0.17}{w / c+0.32}
$$

Cement paste with $0.09 \%$ of MWCNTs were prepared at five water-to-cement ratios $(0.4,0.5$, $0.6,0.7$, and 0.8 ) and the effective electrical conductivity of the dry samples were measured using the method discussed in Section 3.2. Figure 6 shows a comparison of the various micromechanics based models with our experimental results. Note that the electrical conductivity of air voids $\left(\sigma_{a}\right)$ were assumed to be perfectly insulting inclusions as $\sigma_{a}=1.0 \times 10^{-15} \mathrm{~S} / \mathrm{m}$ that 
is the similar value of polymer [41] and the electrical conductivity of the cement hydrates $\left(\sigma_{c}\right)$ was obtained as $\sigma_{c}=2.0 \times 10^{-5} \mathrm{~S} / \mathrm{m}$ by the curve-fitting with experimental data due to a lack of data for pure cement hydrate. It can be seen that the electrical conductivity of cement paste decreases with an increase in the water-cement ratio (porosity). All models provide fairly consistent predictions with the experimental results, but the MGM performs the best. The micropores in cement paste generally are discrete spheres in the cement hydrate, which agree with the assumptions of the MGM model very well. Therefore, the model provides excellent predictions of the experiments.

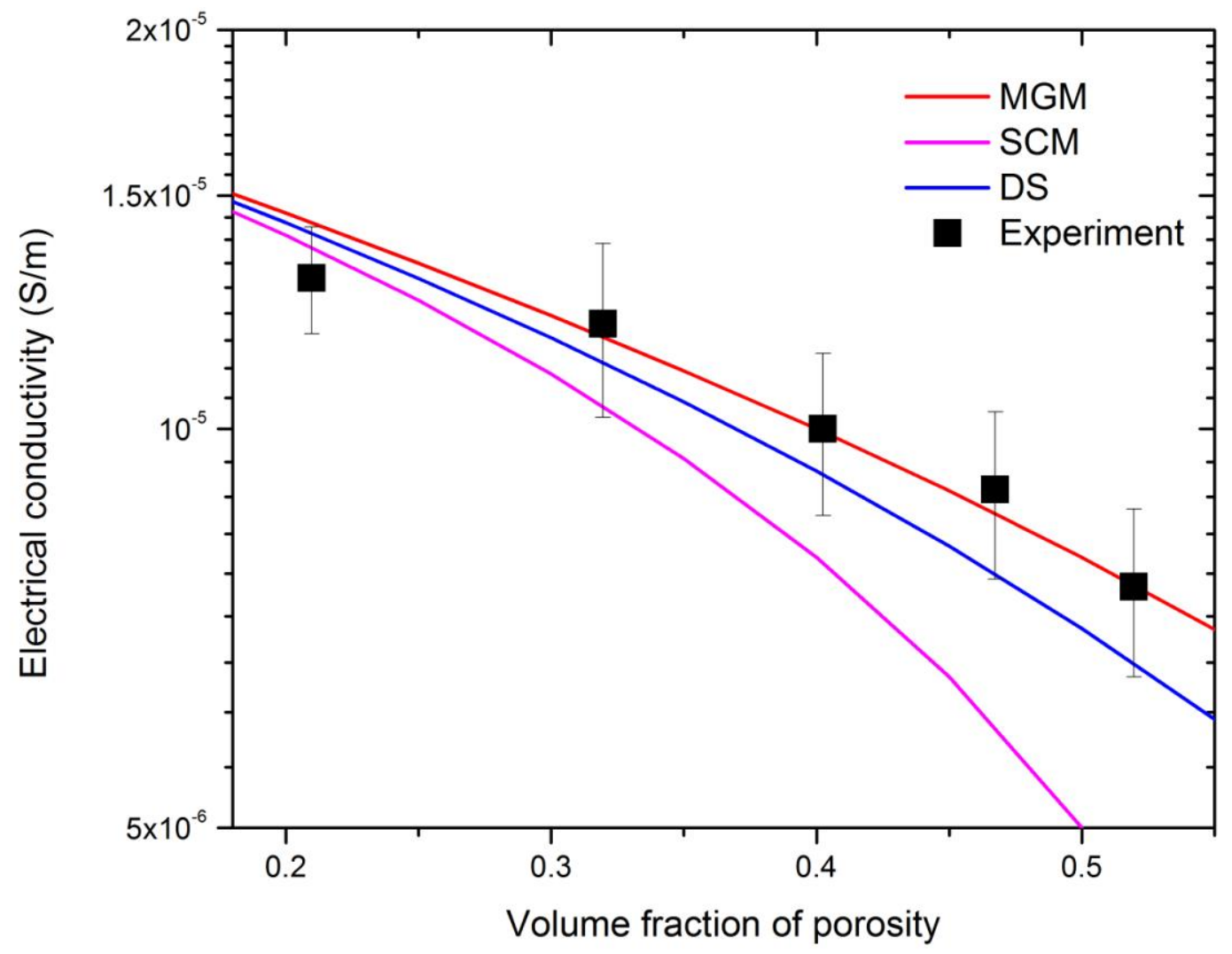

Figure 6. Comparison of various models with experimental results for dry cement paste 


\subsection{Influence of moisture in cement paste}

Moisture is a significant factor that affects the electrical conductivity of cementitious-based composites because the electrical conductivity of moisture is much higher than the solid phases in cement paste. Here, we simulate the effective electrical conductivity of cement paste with 0.09 $\%$ of MWCNTs at a water-to-cement ratio of 0.5 with different volume fractions of moisture ( $\phi_{m}$ $=0 \%, 11 \%, 19 \%, 26 \%, 32 \%$, and $39 \%$ by cement volume). The electrical conductivity of water is approximately $\sigma_{w}=10^{-3} \mathrm{~S} / \mathrm{m}$ when it contains some minerals or when ions from the samples are leached into water. Figure 8 shows the effective electrical conductivity of the composite using various models. All models except for the parallel, series, and SCM provide good predictions compared to the experimental results. In particular, GSCM and MGM are the best models to simulate the effect of moisture in MWCNT/cement paste. Therefore, the MGM can capture the effect of the porosity and moisture on the effective electrical conductivity of the cement paste. 


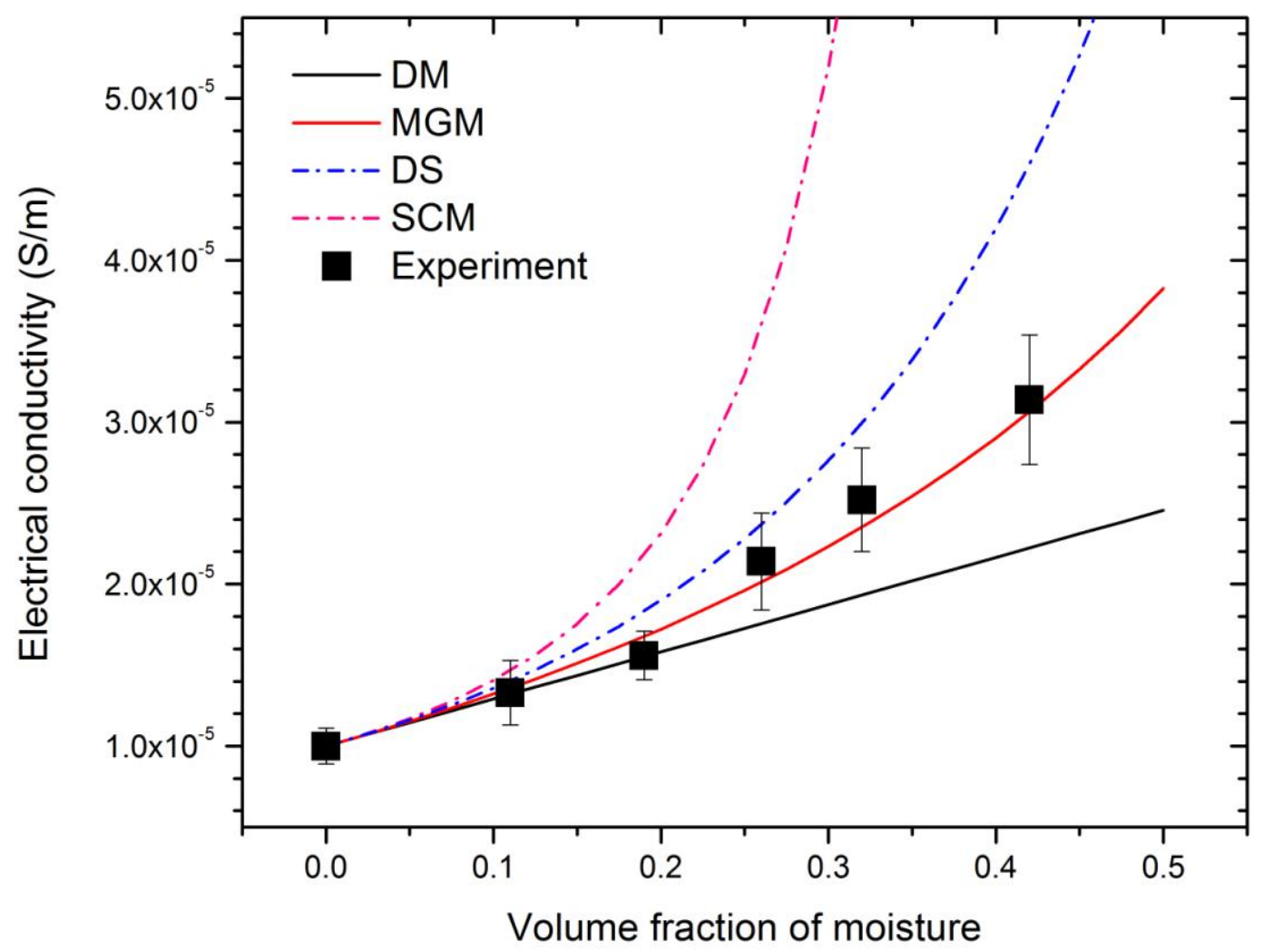

Figure 7. Influence of moisture on electrical conductivity of MWCNT/cement paste

\subsection{Influence of MWCNT}

The electrical conductivity of MWCNT is generally about $10^{6} \mathrm{~S} / \mathrm{m}$ but it has been reported that the electrical conductivity can be lower in the actual application, say in the range of $10^{4} \sim 10^{5} \mathrm{~S} / \mathrm{m}$ [42], due to damage from the fabrication procedure. The electrical conductivity of cement paste was obtained from our experimental results. Six samples with a water-to-cement ratio of 0.5 were prepared at different volume fractions of MWCNTs $(0 \%, 0.09 \%, 0.17 \%, 0.43 \%$, $0.85 \%$, and $1.69 \%)$. The electrical conductivity of the MWCNTs was taken as $\sigma_{C N T}=1.0 \times 10^{4} \mathrm{~S} / \mathrm{m}$. Figure 8 displays the predictions for the micro-mechanical based models, which shows that none of the models provide a good prediction of the experimental results. 
Except for the parallel one, all other models significantly underestimate the effective electrical conductivity of the composites. This is attributed to the fact that those models do not consider the connectivity amongst MWCNTs. In addition, the higher electrical conductivity of MWCNT/cement paste compared to pure cement paste is due to the high aspect ratio of MWCNTs, making contact points between MWCNTs that act as electron paths for better electrical conductivity.

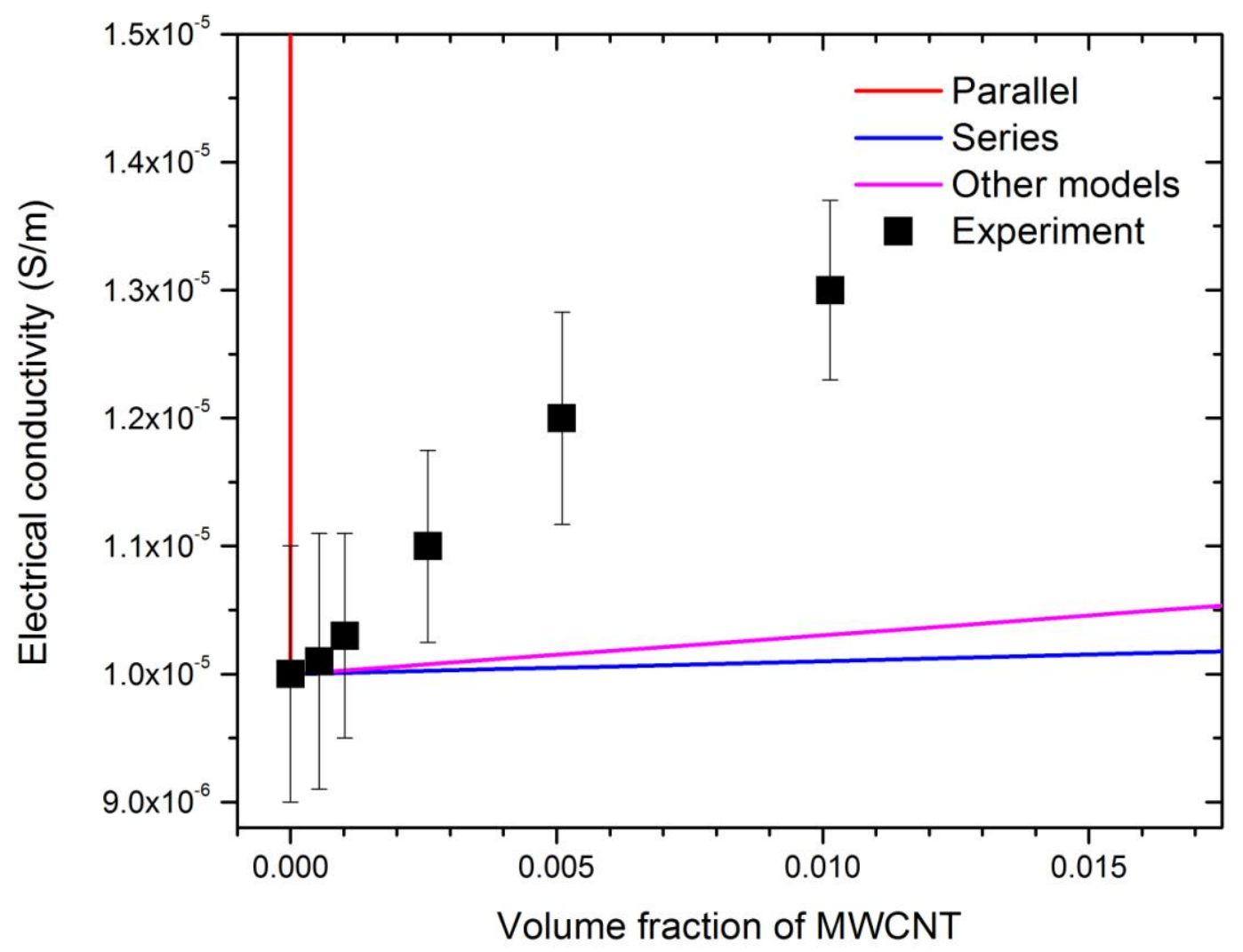

Figure 8. Comparison of measured and predicted electrical conductivity of MWCNT/cement paste 
To capture the effect of electron paths through the MWCNT, we have developed the eight-chain model that is capable to predict the effective electrical conductivity of the composites with wellnetworked multi-walled carbon nanotubes [43]. However, we have observed that the MWCNT is commonly agglomerated in cement paste with a certain portion contributing to the network. Therefore, in this study, we proposed a mixed model to simulate overall electrical conductivity of MWCNT/cement paste. For fully dispersed MWCNT in cement matrix, the eight-chain model was used and will be elaborated in the next subsection. For agglomeration of MWCNTs in the matrix, MGM in Equation (33) was used as it exhibits excellent accuracy for the discrete particles in a continuous matrix. Therefore, the electric conductivity will be the sum of the two parts:

$$
\sigma_{e f f}=\phi_{m i x} \sigma_{\text {network }}+\left(1-\phi_{\text {mix }}\right) \sigma_{\text {agg }}
$$

where $\phi_{\text {mix }}=\eta \phi_{C N T}$ and $\eta$ is a degree of fully dispersed MWCNT from the total volume fraction $\phi_{C N T}$, which decreases with the increase of the volume fraction of MWCNT as the same dispersion effort. For simplicity, a linear function is assumed as $\eta=k_{0}-k_{1} \phi_{C N T}$ with $k_{0}$ and $k_{1}$ being the fitting parameter to describe dispersion effort. Therefore we can write

$$
\phi_{\text {mix }}=\left(k_{0}-k_{1} \phi_{C N T}\right) \phi_{C N T}
$$

Figure 9 shows the effective electrical conductivity of MWCNT/cement paste based on the mixed model. The result highly depends on the degree of well-dispersed MWCNTs. In this study, we obtained good agreement with experimental data when dispersed state was used as $\eta=0.000004-0.000284 \phi$, meaning that severe agglomerations of MWCNTs exist, which significantly lower the electrical conductivity compared to composites consisting of a fullnetworked CNT/polymer composites [43-45]. Note that when the volume fraction of the MWCNTs is high, say $1.0 \%$ in Figure 9, the experimental result is much lower than the prediction due to severe agglomeration of MWCNTs, which has been commonly found in the literature $[39,46]$. 


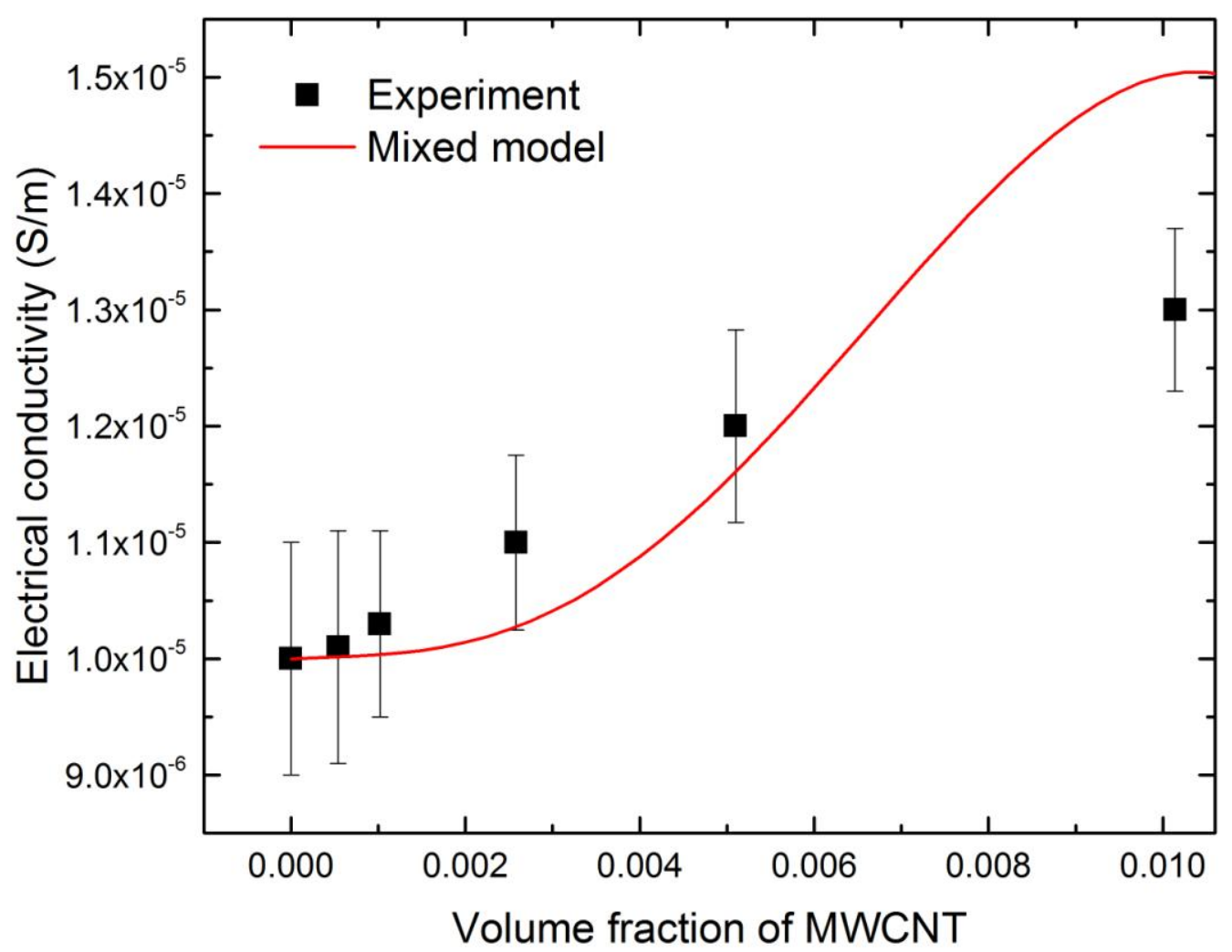

Figure 9. Mixed model for effective electrical conductivity MWCNT/cement paste

\subsection{Prediction of MWCNT/cement composites}

It has been observed that the effective electrical conductivity of MWCNT/cement composite depends on air voids, MWCNTs, and moisture. Two-phase based micromechanical models, in particular, MGM, predict the electrical conductivity of MWCNT/cement composites at various water-to-cement ratios and amounts of moisture. On the other hand, a mixed model constructed from the eight-chain model and the MGM provided good predictions for the addition of MWCNTs to the cement composite. When multiple material phases are considered, one can simulate cement paste prepared at different porosities and moisture contents and MWCNT contents by using the combination of the three steps, which are corresponding to the above three 
cases. Given a MWCNT/cement composite with the porosity $\phi_{p}$, moisture content $\phi_{m}$, and MWCNT content $\phi_{C N T}$, if the dispersion effort has been well described by the paramaters $k_{0}$ and $k_{1}$, the effective electric conductivity can be calculated by the following procedure:

First, the effective electric conductivity of dry cement paste with porosity $\phi_{p}$ is calculated by the MGM model

$$
\sigma^{1}=\sigma_{c} \frac{\left(\sigma_{a}+2 \sigma_{c}\right)+2 \phi_{p}\left(\sigma_{a}-\sigma_{c}\right)}{\left(\sigma_{a}+2 \sigma_{c}\right)-\phi_{p}\left(\sigma_{a}-\sigma_{c}\right)}
$$

Then the effect of moisture will be evaluated by the MGM model again as

$$
\sigma^{2}=\sigma^{1} \frac{\left(\sigma_{w}+2 \sigma^{1}\right)+2 \phi_{m}\left(\sigma_{w}-\sigma^{1}\right)}{\left(\sigma_{w}+2 \sigma^{1}\right)-\phi_{m}\left(\sigma_{w}-\sigma^{1}\right)}
$$

At the last step, the MWCNTs will be considered by the mixed model in Eq. (34) by taking the cement paste with $\sigma^{2}$ as the matrix. Therefore, we obtained the electrical conductivity of agglomerated and networked MWCNTs such as

$$
\begin{gathered}
\sigma_{a g g}=\sigma^{2} \frac{\left(\sigma_{C N T}+2 \sigma^{2}\right)+2 \phi_{C N T}\left(1-\phi_{m i x}\right)\left(\sigma_{w}-\sigma^{2}\right)}{\left(\sigma_{w}+2 \sigma^{2}\right)-\phi_{C N T}\left(1-\phi_{\text {mix }}\right)\left(\sigma_{w}-\sigma^{2}\right)} \\
\sigma_{\text {network }}=\left\{\left(\frac{\sqrt{3} \pi D^{2}}{\phi_{C N T}}\right)^{1 / 2} \cdot R_{\text {eff }}\right\}^{-1}
\end{gathered}
$$

where $D$ is a diameter of MWCNT and $R_{\text {eff }}$ is total resistance in a unit cell which consisting of an intrinsic resistance of MWCNT and a contact resistance between MWCNTs. All of the parameters that were used are the same as given by Jang and Yin [43]. Eventually, we can use the results of Eqs. (38) and (39) in Eq. (34) to obtain the effective electrical conductivity of the composites. Figure 10 shows a relationship between measured and predicted electrical conductivity of cementitious-based composite with moisture and MWCNTs. The proposed model provides very consistent predictions with the experimental results and the overall root 
mean squared error (RMSE) is 0.0866 , which is $~ 5 \%$ of the average electrical conductivity of all samples. Adding MWCNTs can improve the electrical conductivity significantly but the moisture exhibits a more significant effect on the overall conductivity. Therefore, it should be very cautions to use MWCNT/cementitious-based composites for sensing with electrical conductivity change because the environmental factor of moisture may play a dominant role in the measurement. The proposed model can successfully capture the effect of both MWCNTs and moisture.

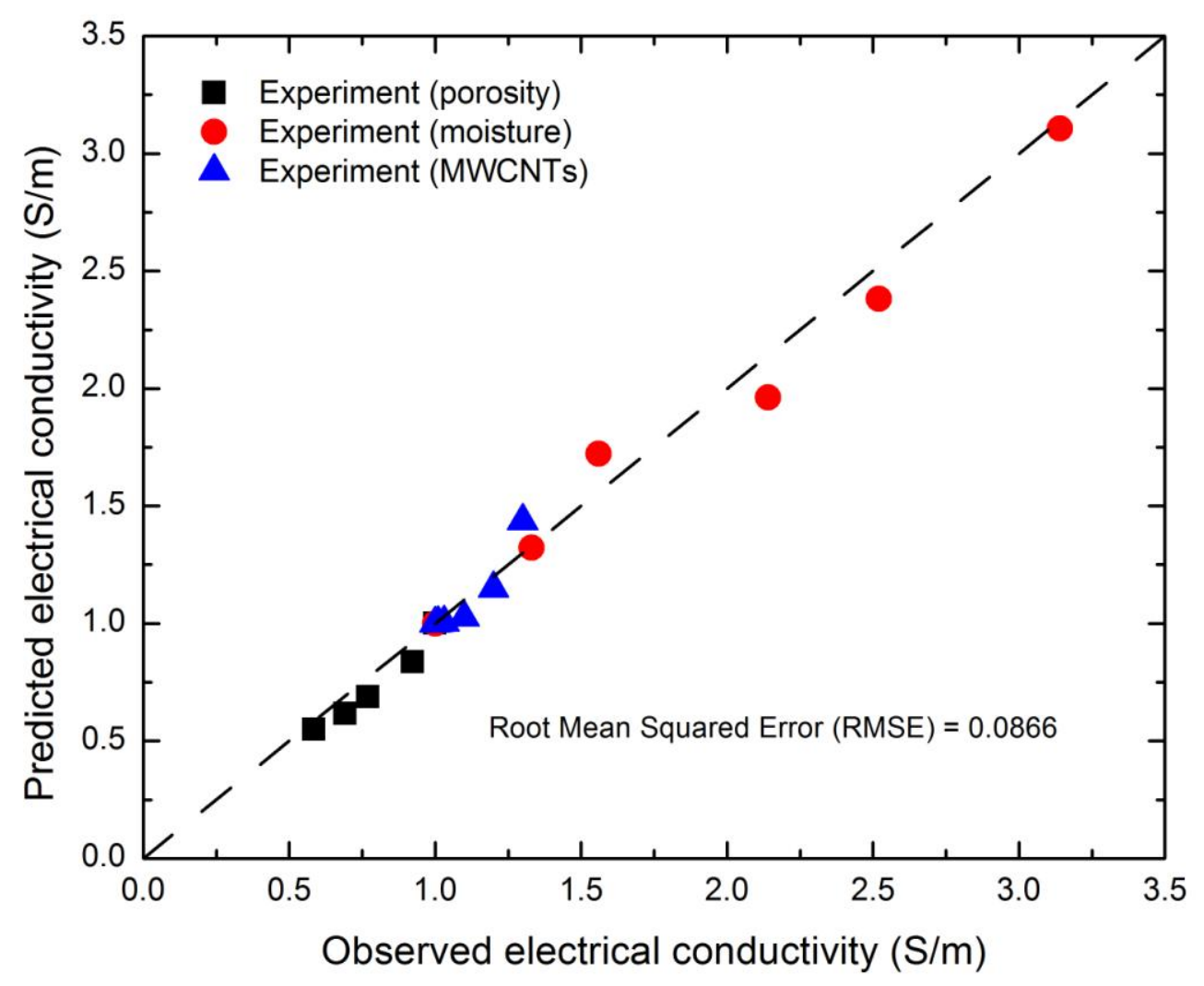

Figure 10. Relationship between the observed and predicted electrical conductivity in terms of porosity, moisture, and MWCNTs 
Notice that adding MWCNTs may change the effective porosity and moisture content as it changes the overall volume of the composite in principle. However, because the MWCNT content is always small ( $1 \%)$, the difference between two cases is negligible. Therefore, we can use the same input parameters although in the first two steps, the volume fractions should be calculated based on the cement paste without MWCNTs.

\section{Conclusions}

Several micromechanical models for effective electrical conductivity of cementitious-based composite with air void, MWCNT, and moisture are reviewed and examined through the analytical derivation and comparison with our experiments. The understanding can be extended to other physical properties of cementitious-based composite such as thermal conductivity. The main conclusions are provided as follows:

(1) The MWCNT agglomerations were observed in MWCNT/cement paste due to the polarity of water and alkalinity of cement, significantly reducing its electrical conductivity and thus affecting the accuracy of conventional micromechanics.

(2) The MGM model provided excellent accuracy to evaluate the effect of discrete particle phase, such as air voids and moisture. However, all micromechanical models failed to capture the effect of electron path through the MWCNT network on the electrical conductivity of the MWCNT/cement paste due to MWCNT agglomerations.

(3) The mixed model based on the eight-chain model for MWCNT network and MGM for MWCNT agglomeration was proposed to simulate the effect of MWCNT agglomeration in the MWCNT/cement pastes.

(4) A three-step micromechanical model was proposed to predict the effective electrical conductivity of MWCNT/cement pastes. The proposed model provided very good agreement with experimental results with an overall root mean squared error (RMSE) at 0.0866, which is $\sim 5 \%$ of the average electrical conductivity of all samples. The research results showed that moisture may significantly affect the accuracy of the sensing technology with smart cementitious-based composites unless this factor is well quantified. 


\section{Acknowledgements}

The authors would like to thank Dr. Liming Li and Dr. Fangliang Chen for their valuable assistance with the experiments. This work is sponsored by the National Science Foundation CMMI 1301288, whose support is gratefully acknowledged.

\section{References}

[1] M. S. Konsta-Gdoutos, Z. S. Metaxa, and S. P. Shah, "Highly dispersed carbon nanotube reinforced cement based materials," Cement and Concrete Research, vol. 40, pp. 10521059, Jul 2010.

[2] T. Nochaiya and A. Chaipanich, "Behavior of multi-walled carbon nanotubes on the porosity and microstructure of cement-based materials," Applied Surface Science, vol. 257, pp. 1941-1945, Jan 12011.

[3] R. K. Abu Al-Rub, A. I. Ashour, and B. M. Tyson, "On the aspect ratio effect of multiwalled carbon nanotube reinforcements on the mechanical properties of cementitious nanocomposites," Construction and Building Materials, vol. 35, pp. 647-655, Oct 2012.

[4] Y. Hu, D. N. Luo, P. H. Li, Q. B. Li, and G. Q. Sun, "Fracture toughness enhancement of cement paste with multi-walled carbon nanotubes," Construction and Building Materials, vol. 70, pp. 332-338, Nov 152014.

[5] S. H. Wen and D. D. L. Chung, "Electrical-resistance-based damage self-sensing in carbon fiber reinforced cement," Carbon, vol. 45, pp. 710-716, Apr 2007.

[6] H. K. Kim, I. W. Nam, and H. K. Lee, "Enhanced effect of carbon nanotube on mechanical and electrical properties of cement composites by incorporation of silica fume," Composite Structures, vol. 107, pp. 60-69, Jan 2014.

[7] H. Li, Q. Q. Zhang, and H. G. Xiao, "Self-deicing road system with a CNFP highefficiency thermal source and MWCNT/cement-based high-thermal conductive composites," Cold Regions Science and Technology, vol. 86, pp. 22-35, Feb 2013. 
[8] H. Li, Q. Q. Zhang, and H. G. Xiao, "Analytic investigations of CNFP-based self-deicing road system on the deicing performance," Cold Regions Science and Technology, vol. 103, pp. 123-132, Jul 2014.

[9] S. H. Wen and D. D. L. Chung, "Partial. replacement of carbon fiber by carbon black in multifunctional cement-matrix composites," Carbon, vol. 45, pp. 505-513, Mar 2007.

[10] F. Azhari and N. Banthia, "Cement-based sensors with carbon fibers and carbon nanotubes for piezoresistive sensing," Cement \& Concrete Composites, vol. 34, pp. 866873, Aug 2012.

[11] B. G. Han, X. Yu, and E. Kwon, "A self-sensing carbon nanotube/cement composite for traffic monitoring," Nanotechnology, vol. 20, Nov 42009.

[12] F. Ubertini, A. L. Materazzi, A. D'Alessandro, and S. Laflamme, "Natural frequencies identification of a reinforced concrete beam using carbon nanotube cement-based sensors," Engineering Structures, vol. 60, pp. 265-275, Feb 2014.

[13] S. H. Jang and H. M. Yin, "Effect of aligned ferromagnetic particles on strain sensitivity of multi-walled carbon nanotube/polydimethylsiloxane sensors," Applied Physics Letters, vol. 106, Apr 62015.

[14] R. Sriravindrarajah, R. Swamy, R. Sriravindrarajah, and R. Swamy, "Development of a Conductivity Probe to Monitor Setting Time and Moisture Movement in Concrete," 1982.

[15] G. Woelfl, K. Lauer, G. Woelfl, and K. Lauer, "The Electrical Resistivity of Concrete with Emphasis on the Use of Electrical Resistance for Measuring Moisture Content," 1979.

[16] A. Princigallo, K. van Breugel, and G. Levita, "Influence of the aggregate on the electrical conductivity of Portland cement concretes," Cement and Concrete Research, vol. 33, pp. 1755-1763, Nov 2003.

[17] N. Schwarz, M. DuBois, and N. Neithalath, "Electrical conductivity based characterization of plain and coarse glass powder modified cement pastes," Cement \& Concrete Composites, vol. 29, pp. 656-666, Oct 2007.

[18] Z. Y. Liu, Y. S. Zhang, L. B. Liu, and Q. Jiang, "An analytical model for determining the relative electrical resistivity of cement paste and C-S-H gel," Construction and Building Materials, vol. 48, pp. 647-655, Nov 2013. 
[19] M. Saleem, M. Shameem, S. E. Hussain, and M. Maslehuddin, "Effect of moisture, chloride and sulphate contamination on the electrical resistivity of Portland cement concrete," Construction and Building Materials, vol. 10, pp. 209-214, Apr 1996.

[20] H. K. Kim, I. S. Park, and H. K. Lee, "Improved piezoresistive sensitivity and stability of CNT/cement mortar composites with low water-binder ratio," Composite Structures, vol. 116, pp. 713-719, Sep-Oct 2014.

[21] B. Sareni, L. Krahenbuhl, A. Beroual, and C. Brosseau, "Effective dielectric constant of random composite materials," Journal of Applied Physics, vol. 81, pp. 2375-2383, Mar 1 1997.

[22] J. C. M. Garnett, "Colours in metal glasses and in metallic films.," Philosophical Transactions of the Royal Society of London Series a-Containing Papers of a Mathematical or Physical Character, vol. 203, pp. 385-420, Sep 1904.

[23] R. Landauer, "Electrical conductivity in inhomogeneous media," AIP Conference Proceedings, vol. 40, pp. 2-45, 1978.

[24] T. J. Fiske, H. S. Gokturk, and D. M. Kalyon, "Percolation in magnetic composites," Journal of Materials Science, vol. 32, pp. 5551-5560, 1997// 1997.

[25] R. Pelster, "Dielectric spectroscopy of confinement effects in polar materials," Physical Review B, vol. 59, pp. 9214-9228, Apr 11999.

[26] H. M. Yin, G. H. Paulino, W. G. Buttlar, and L. Z. Sun, "Effective thermal conductivity of two-phase functionally graded particulate composites," Journal of Applied Physics, vol. 98, Sep 152005.

[27] H. M. Yin, G. H. Paulino, W. G. Buttlar, and L. Z. Sun, "Effective thermal conductivity of functionally graded particulate nanocomposites with interfacial thermal resistance," Journal of Applied Mechanics-Transactions of the Asme, vol. 75, Sep 2008.

[28] H. M. Yin, W. G. Buttlar, G. H. Paulino, and H. Di Benedetto, "Assessment of existing micro-mechanical models for asphalt mastics considering viscoelastic effects," Road Materials and Pavement Design, vol. 9, pp. 31-57, Jan-Mar 2008.

[29] H. M. Yin and B. Lai, "Visco-elastic characterisation of zeolite modified asphalt binder considering phase transformation and air void interaction," Road Materials and Pavement Design, vol. 13, pp. 279-299, 2012. 
[30] H. M. Yin and L. Z. Sun, "Magnetic properties of randomly dispersed magnetic particulate composites: A theoretical study," Physical Review B, vol. 72, Aug 2005.

[31] T. Miloh and Y. Benveniste, "A Generalized Self-Consistent Method for the Effective Conductivity of Composites with Ellipsoidal Inclusions and Cracked Bodies," Journal of Applied Physics, vol. 63, pp. 789-796, Feb 11988.

[32] Z. Hashin and S. Shtrikman, "A Variational Approach to the Theory of the Elastic Behaviour of Polycrystals," Journal of the Mechanics and Physics of Solids, vol. 10, pp. 343-352, 1962.

[33] J. K. Lee and J. G. Kim, "Derivation of governing equation for predicting thermal conductivity of composites with spherical inclusions and its applications," Physics Letters A, vol. 375, pp. 3739-3744, Oct 32011.

[34] S. Musso, J. M. Tulliani, G. Ferro, and A. Tagliaferro, "Influence of carbon nanotubes structure on the mechanical behavior of cement composites," Composites Science and Technology, vol. 69, pp. 1985-1990, Sep 2009.

[35] L. Vaisman, H. D. Wagner, and G. Marom, "The role of surfactants in dispersion of carbon nanotubes," Advances in Colloid and Interface Science, vol. 128, pp. 37-46, Dec 212006.

[36] S. H. Wen and D. D. L. Chung, "Effect of admixtures on the dielectric constant of cement paste," Cement and Concrete Research, vol. 31, pp. 673-677, Apr 2001.

[37] D. J. Yang, C. G. Lu, H. M. Yin, and I. P. Herman, "Thermoelectric performance of PbSe quantum dot films," Nanoscale, vol. 5, pp. 7290-7296, 2013.

[38] K. Ke, Y. Wang, Y. Luo, W. Yang, B. H. Xie, and M. B. Yang, "Evolution of agglomerate structure of carbon nanotubes in multi-walled carbon nanotubes/polymer composite melt: A rheo-electrical study," Composites Part B-Engineering, vol. 43, pp. 3281-3287, Dec 2012.

[39] O. Mendoza, G. Sierra, and J. I. Tobon, "Effect of the reagglomeration process of multiwalled carbon nanotubes dispersions on the early activity of nanosilica in cement composites," Construction and Building Materials, vol. 54, pp. 550-557, Mar 152014.

[40] T. C. Hansen, "Physical structure of hardened cement paste. A classical approach," Materials and Structures, vol. 19, pp. 423-436, 1986// 1986. 
[41] H. S. Wong, A. M. Pappas, R. W. Zimmerman, and N. R. Buenfeld, "Effect of entrained air voids on the microstructure and mass transport properties of concrete," Cement and Concrete Research, vol. 41, pp. 1067-1077, Oct 2011.

[42] W. S. Bao, S. A. Meguid, Z. H. Zhu, and M. J. Meguid, "Modeling electrical conductivities of nanocomposites with aligned carbon nanotubes," Nanotechnology, vol. 22, Dec 2011.

[43] S. H. Jang and H. M. Yin, "Effective electrical conductivity of carbon nanotube-polymer composites: a simplified model and its validation," Materials Research Express, vol. 2, Apr 2015.

[44] F. H. Gojny, M. H. G. Wichmann, B. Fiedler, I. A. Kinloch, W. Bauhofer, A. H. Windle, et al., "Evaluation and identification of electrical and thermal conduction mechanisms in carbon nanotube/epoxy composites," Polymer, vol. 47, pp. 2036-2045, Mar 82006.

[45] S. Farjana, F. Toomadj, P. Lundgren, A. Sanz-Velasco, O. Naboka, and P. Enoksson, "Conductivity-Dependent Strain Response of Carbon Nanotube Treated Bacterial Nanocellulose," Journal of Sensors, 2013.

[46] S. H. Jang, S. Kawashima, and H. M. Yin, "Influence of Carbon Nanotube Clustering on Mechanical and Electrical Properties of Cement Pastes," Materials, vol. 9, Apr 2016. 